\title{
Enhancing the oral bioavailability of biochanin A by encapsulation in mixed micelles containing Pluronic FI27 and Plasdone S630
}

This article was published in the following Dove Press journal:

International Journal of Nanomedicine

22 February 2017

Number of times this article has been viewed

\author{
Xiaoyan $\mathrm{Wu}^{\prime}$ \\ Weihong $\mathrm{Ge}^{\prime}$ \\ Tengfei Shao' \\ Weijun $\mathrm{Wu}^{\prime}$ \\ Jian $\mathrm{Hou}^{2}$ \\ Li Cui ${ }^{2}$ \\ Jing Wang ${ }^{2}$ \\ Zhenghai Zhang ${ }^{2}$ \\ 'Department of Pharmacy, The \\ Affiliated Nanjing Drum Tower \\ Hospital of Nanjing University Medical \\ School, ${ }^{2}$ Key Laboratory of New Drug \\ Delivery System of Chinese Materia \\ Medica, Jiangsu Provincial Academy \\ of Chinese Medicine, Nanjing, Jiangsu, \\ People's Republic of China
}

Correspondence: Jing Wang Key Laboratory of New Drug Delivery System of Chinese Materia Medica, Jiangsu Provincial Academy of Chinese Medicine, 100 Shizi Road, Nanjing, Jiangsu 210028, People's Republic of China

Tel $+862513675 \mid 23890$

Fax +862552362115

Email wangjing3968@126.com
Abstract: Biochanin A (BCA), a natural dietary isoflavone, has been reported to show anticancer activities. However, its low biological availability and poor aqueous solubility limit its usefulness as a chemotherapeutic agent. We developed BCA-loaded micelles with Pluronic F127 and Plasdone S630 (BCA-FS). The optimized, spherical-shaped BCA-FS was obtained at a ratio of 1:1 (F127:S630). The particle size was $25.17 \pm 1.2 \mathrm{~nm}$, and the zeta potential was $-10.9 \pm 0.24 \mathrm{mV}$. BCA solubility in water increased to $5.0 \mathrm{mg} / \mathrm{mL}$ after encapsulation, and the drug-loading efficiency was $5.88 \% \pm 0.76 \%$. In vitro release experiments showed a delayed release of BCA from the mixed micelles. Furthermore, the BCA absorption permeability across a Caco- 2 cell monolayer from the apical side to the basolateral side increased by $54 \%$ in BCA-FS. A pharmacokinetics evaluation showed a 2.16-fold increase in the relative oral bioavailability of BCA-FS compared with raw BCA, indicating that the mixed micelles may promote absorption in the gastrointestinal tract. A gastrointestinal safety assay was used to assess the reliability and safety of BCA-FS. On the basis of these findings, we conclude that this simple nanomicelle system could be leveraged to deliver BCA and other hydrophobic drugs.

Keywords: biochanin A, mixed micelles, oral bioavailability, Pluronic F127, Plasdone S630

\section{Introduction}

Isoflavone-rich foods and dietary supplements are garnering interest as agents for disease chemoprevention. Biochanin A (BCA; Figure 1A), a dietary isoflavone found in red clover, cabbage, and alfalfa, has been associated with various health benefits. As an anticancer compound, BCA was shown to be effective in reducing prostate, ${ }^{1}$ breast, ${ }^{2}$ and pancreatic ${ }^{3}$ cancer cell survival by acting on the cell proliferation and apoptosis pathways. In addition, owing to the structural similarities between BCA and estrogen, BCA can exert both estrogenic and anti-estrogenic effects ${ }^{4}$ and is marketed for the treatment of inflammation, ${ }^{5,6}$ osteoporosis, ${ }^{7,8}$ and other diseases. ${ }^{910}$ However, its clinical efficacy is limited by its low oral bioavailability, which is due to poor water solubility, high clearance, and large apparent volume of distribution. ${ }^{11}$ Hence, new delivery systems are required to improve the performance of BCA.

Nanostructured lipid carriers (NLCs) have been synthesized to improve the poor aqueous solubility and bioavailability of BCA. ${ }^{12}$ These NLCs are modified with polyethylene glycol (PEG) to enhance the surface hydrophilicity; the PEG chains are designed to reduce charge-based contact typical of proteins and small-molecule interactions. ${ }^{13}$ In general, NLCs are designed to be biodegradable and biocompatible. Nonetheless, they possess some limitations, such as manufacturing complexities and diseconomy in 
A

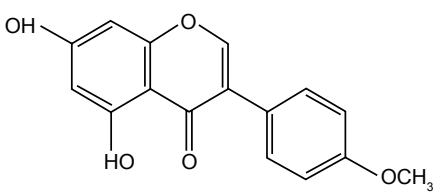

B

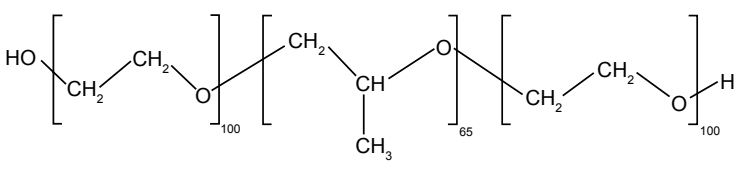

C

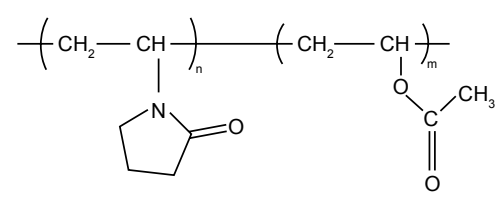

Figure I Chemical structure of drug and excipients.

Notes: (A) Structure of biochanin A; (B) structure of Pluronic FI27; and (C) structure of Plasdone S630.

industrial production. Self-assembled micelle systems have also been shown to be a promising technique for improving delivery of poorly soluble drugs; ${ }^{14,15}$ the advantages of these systems include controlled drug release, enhanced drug solubility, and no immune response. ${ }^{16}$ Furthermore, the simple mechanism of formation is easy to apply during the manufacturing process.

In our design, a novel binary mixture of micelles was established using a self-assembly method by using Pluronic F127 (F127, Figure 1B) and Plasdone ${ }^{\circledR}$ S630 (S630, Figure 1C); our aim was to overcome the challenges of BCA's poor oral bioavailability. The binary mixture shows favorable characteristics compared with a single-material system. Pluronic F127 consists of polyoxyethylene (PEO) units (70\%) and polyoxypropylene (PPO) blocks (30\%) and has a versatile triblock composition of PEO100-PPO65-PEO100. The hydrophobic PPO segments can form a hydrophobic core spontaneously, where hydrophobic drugs can be incorporated into the microenvironment. F127 is widely used in nanoparticle preparations because of its safety, long circulation period, and biocompatibility. However, its use is limited by its poor solubility and low stability, which make it difficult to use alone. ${ }^{17-19}$ Plasdone S630 is a water-soluble organic polymer composed of polyvinylpyrrolidone (PVP) and vinyl acetate (VA), and has been widely applied in solid-dispersion formulations. ${ }^{20-22}$ Because of the copolymerization, Plasdone S630 retains the merits of PVP, such as good solubility in water, good film formation, and better bonding performance, and incorporates extensive drug solubilization capacity and better surface activity derived from VA. Therefore, based on our previous studies, ${ }^{23,24}$ we speculated that the new combination of binary mixed micelles could enhance the aqueous solubility of BCA and improve its oral bioavailability.
In this study, BCA-loaded micelles with Pluronic F127 and Plasdone S630 (BCA-FS) were prepared by a simple self-assembly method. To evaluate the feasibility of the drug delivery system, we investigated the physicochemical properties of BCA-FS in vitro, including morphology, particle size, zeta potential, drug-loading capacity, and drug release. We also evaluated the absorption and efflux characteristics of BCA-FS in Caco-2 cell monolayers. Pharmacokinetics and gastrointestinal safety were assessed in vivo.

\section{Materials and methods Materials}

BCA (98\%) was purchased from Aladdin (Shanghai, People's Republic of China). Plasdone S630 was obtained from Ashland (Covington, KY, USA). Pluronic F127 was provided by Sigma-Aldrich (St Louis, MO, USA). Caco-2 TC7 cells were a kind gift from Dr Ming Hu of INSERM U178 (Houston, TX, USA). Milli-Q water (Millipore, Bedford, MA, USA) was used throughout the study. Acetonitrile and methanol were obtained from Merck (Darmstadt, Germany). Tween 80 was obtained from Sinopharm Chemical Reagent Co., Ltd (Shanghai, People's Republic of China). All other chemicals were of analytical grade and were obtained from Tedia (Fairfield, OH, USA).

\section{Animals}

Sprague Dawley (SD) rats were obtained from the Experimental Animal Center of Shanghai (SLAS Lab, Shanghai, People's Republic of China) and used for the pharmacokinetics study. Rats had access to food and water ad libitum, and were housed at a temperature of $25^{\circ} \mathrm{C}$ and relative humidity of $45 \%$ for 2 weeks. The use of these animals and cell lines were carried out in accordance with the guidelines of the Guide for the Care and Use of Laboratory Animals, which was evaluated and approved by the Institutional Ethics Committee of Jiangsu Provincial Academy of Chinese Medicine, who approved the overall study.

\section{Preparation of BCA-FS micelles}

BCA-loaded mixed micelles were prepared according to a self-assembly technique as previously described. ${ }^{23}$ In brief, BCA was dissolved in ethanol at a concentration of $5 \mathrm{mg} / \mathrm{mL}$. F127 and S630 were combined in different ratios and dispersed in ethanol at $45^{\circ} \mathrm{C}$. The $\mathrm{F} 127 / \mathrm{S} 630$ ethanol solution was stirred uniformly until it reached equilibrium. The BCA ethanol solution was then added dropwise into the F127/S630 solution with magnetic stirring. After $1 \mathrm{~h}$ of gentle stirring at $25^{\circ} \mathrm{C}$, the ethanol was removed under reduced pressure at $45^{\circ} \mathrm{C}$. Deionized water was added to the flask, and a transparent solution formed after shaking. The mixed micelles 
were then acquired after ultracentrifugation at 14,000 rpm $(18.8 \times g)$ for $10 \mathrm{~min}$. Finally, the supernatant was retained and the stability of the mixed micelles was assessed.

\section{Characteristics of BCA-FS micelles}

Particle size and zeta potential analysis

Mean particle size, polydispersity index (PDI), and zeta potential of BCA-FS in the dispersion were determined in triplicate by dynamic light scattering using a Nano ZS system (Malvern Instruments, Malvern, UK) at $25^{\circ} \mathrm{C}$. The mixed micelles were diluted fivefold with water for injection before measurement. Zeta potential measurement was carried out using a zeta potential analyzer.

\section{Transmission electron microscopy}

BCA-FS morphology was observed using transmission electron microscopy (TEM, JEM-200CX; Jeol, Tokyo, Japan). BCA-FS droplets were applied to a copper grid and then stained with $2 \%(\mathrm{w} / \mathrm{v})$ phosphotungstic acid for $30 \mathrm{~s}$. The grid was observed using TEM after infrared drying for $5 \mathrm{~min}$.

\section{Determination of drug loading}

Drug loading was determined by high-performance liquid chromatography (HPLC), which consisted of a pump (G1311G; Agilent Technologies, Palo Alto, CA, USA), a UV detector (G4212B; Agilent Technologies) set at $260 \mathrm{~nm}$, an autosampler (G1329B; Agilent Technologies), and a reversed-phase $\mathrm{C}_{18}$ column (4.6×250 mm, $5 \mu \mathrm{m}$, SunFire; Waters Co., Milford, MA, USA). The mobile phase, with a flow rate of $1.0 \mathrm{~mL} / \mathrm{min}$, consisted of a mixture of acetonitrile and $0.1 \%$ phosphoric acid solution $(50: 50, \mathrm{v} / \mathrm{v})$, which was freshly prepared and filtered with a $0.45 \mu \mathrm{m}$ membrane. BCA-loaded dried micelles were obtained after freeze-drying. Weighed amounts of dried micelles were then dissolved in methanol and fragmented by ultrasonication. The entrapment efficiency (EE) and drug-loading (DL) content of $\mathrm{BCA}$ were calculated using the following equations:

$$
\mathrm{EE}(\%)=\frac{\text { Weight of BCA in polymeric micelles }}{\text { Weight of BCA fed initially }} \times 100
$$

$$
\text { DL }(\%)=\frac{\text { Weight of BCA in polymeric micelles }}{\text { Weight of polymeric micelles }} \times 100
$$

\section{In vitro release experiment}

The dialysis bag method was used to estimate the drug release from BCA-FS in vitro; this method retains micelles and allows the free BCA into the dissolution media with a molecular weight cut-off of 3,500 (Greenbird, Inc., Shanghai, People's Republic of China). The bags were soaked in double-distilled water for $12 \mathrm{~h}$ before use. Next, $1 \mathrm{~mL}$ of BCA-FS was poured into the bag and the two ends fixed by clamps. The bags were placed into conical flasks containing the receiving phase, which consisted of $200 \mathrm{~mL}$ phosphatebuffered saline (PBS, pH 6.8$)$ at $37^{\circ} \mathrm{C}$ and $0.5 \%(\mathrm{w} / \mathrm{v})$ Tween 80 . The conical flasks were placed into a thermostatic shaker (HZQ-C; Shuangjie, Changzhou, People's Republic of China) at $37^{\circ} \mathrm{C}$ and shaken at a rate of 140 times per min. At 1, 2, 3, 6, 8, 20, 44, and $72 \mathrm{~h}$ after beginning the test, triplicate suspensions $(0.5 \mathrm{~mL})$ of the release medium were removed and filtered through a $0.45 \mu \mathrm{m}$ membrane for analysis, while fresh dialysis medium was added to maintain sink conditions. The filtrate was analyzed using HPLC at the different time points.

\section{Transport experiments in Caco-2 cell culture model}

The Caco-2 TC7 cell line, similar to wild-type Caco-2 cells, was selected to investigate BCA absorption in this study owing to its stability. Culture conditions for growing Caco-2 cells were the same as in our previous studies. ${ }^{23}$ Briefly, Caco-2 cells were grown in high-glucose Dulbecco's Modified Eagle's Medium at $37^{\circ} \mathrm{C}$ in a $5 \% \mathrm{CO}_{2}$ atmosphere and at a relative humidity of $95 \%$, supplemented with $10 \%$ fetal bovine serum, $1 \%$ minimal essential medium non-essential amino acids, $2 \mathrm{mM}$ L-glutamine, and $1 \%$ penicillin/streptomycin. Cells were seeded at a density of $1 \times 10^{6} \mathrm{cells} / \mathrm{cm}^{2}$ on untreated culture inserts of polycarbonate membranes with $0.4 \mu \mathrm{m}$ pores. Culture inserts containing a Caco-2 monolayer were placed in 12-mm transwell plates. Approximately 21 days after seeding, the cells were fully differentiated and transepithelial electrical resistance (TEER) values became stable $\left(>400 \Omega / \mathrm{cm}^{2}\right)$. Transport studies were not carried out until the cell monolayer was formed..$^{25}$

Caco-2 transport studies were initiated by removing the medium from both sides of the monolayer, followed by replacement with apical (AP) and basolateral (BL) buffers prewarmed to $37^{\circ} \mathrm{C}$. The cell monolayers were incubated in a shock shaker for $30 \mathrm{~min}$ at $37^{\circ} \mathrm{C}(100 \mathrm{cycles} / \mathrm{min})$ until equilibration. After aspirating the incubation medium, a solution of dissolved mixed micelles loaded with $20 \mu \mathrm{M}$ BCA was added to the AP or BL side. At fixed time points $(0,1,2,3$, and $4 \mathrm{~h}$ after incubation), $400-\mu \mathrm{L}$ aliquots were taken from both the donor and receiver sides, and the same volume of donor solution (containing BCA) and transport medium (Hank's balanced salt solution) was replaced after each sampling. One hundred microliters of internal standard 
solution (chloramphenicol [CM] $100 \mu \mathrm{M}$ in methanol) were immediately added to samples to stabilize them until analysis. These samples were then centrifuged at 16,000 rpm for $5 \mathrm{~min}$ at $4^{\circ} \mathrm{C}$, and the supernatant was analyzed using HPLC. TEER values were measured at the end of the transport experiment and compared to the TEER values measured at baseline. The BCA permeability coefficient was calculated from the linear plot of the quantity transported versus time, using the following equation:

$$
P_{\text {app }}=\frac{1}{C_{0} \times S} \times \frac{d Q}{d t}
$$

where $\mathrm{C}_{0}$ is the initial concentration of $\mathrm{BCA}$ on the donor side, $\mathrm{S}$ is the surface area of the cell monolayer $\left(4.2 \mathrm{~cm}^{2}\right)$, and $\mathrm{dQ} / \mathrm{dt}$ is the steady-state appearance rate of BCA on the receiver side.

\section{Gastrointestinal mucosa toxicity test}

SD rats (average weight $220 \mathrm{~g}$ ) were randomly divided into two groups and treated with normal saline (control group) or BCA $(20 \mathrm{mg} / \mathrm{kg})$ three times per week for 1 month. Mice were killed and $1 \mathrm{~cm}$ segments of stomach and small intestine were excised from the gastrointestinal tract. These tissues were fixed in 10\% formaldehyde-based fixative for $24 \mathrm{~h}$, rehydrated, and then embedded into paraffin. Next, $5 \mu \mathrm{m}$ sections of these tissues were stained with hematoxylin and eosin $(\mathrm{H} \& \mathrm{E})$. The samples were imaged with a light microscope.

\section{Pharmacokinetic studies}

Experimental process and sample treatment

For the pharmacokinetic study, ${ }^{26,27} \mathrm{SD}$ rats (weight $220 \pm 10 \mathrm{~g}$ ) were divided randomly into two groups $(n=6)$. All rats were fasted overnight to prevent coprophagia, but had free access to water. The first group received the BCA suspension orally, dispersed in $0.1 \%$ aqueous sodium carboxymethyl cellulose; the second group received $50 \mathrm{mg} / \mathrm{kg}$ of BCA-FS. All oral formulations were diluted with water immediately prior to administration. Blood samples were collected from the retro-orbital plexus in tubes containing heparin at 0,5 , 15 , and $30 \mathrm{~min}$, and at $1,1.5,2,3,4,5,6,8,12$, and $24 \mathrm{~h}$ following treatment. The blood samples were centrifuged for $10 \mathrm{~min}$ at $10,000 \mathrm{rpm}$, and the supernatant plasma fraction was stored at $-80^{\circ} \mathrm{C}$ until ultra-performance liquid chromatography (UPLC) analysis.

The supernatant pretreatment was conducted by mixing $130 \mu \mathrm{L}$ of plasma sample with $20 \mu \mathrm{L}$ of the internal standard
$\mathrm{CM}(13.858 \mathrm{ng} / \mathrm{mL}$ in methanol). After vortexing, the liquidliquid extraction was accomplished by adding $1 \mathrm{~mL}$ of ethyl acetate, followed by gentle agitation for $4 \mathrm{~min}$. The mixture was centrifuged for $5 \mathrm{~min}$ at $8,000 \mathrm{rpm}$, and $800 \mu \mathrm{L}$ of the organic layer was then transferred to a clean vial and evaporated until dry. Finally, the residue was dissolved in $520 \mu \mathrm{L}$ of the initial mobile phase, transferred to autosampler vials, capped, and placed in the UPLC autosampler. Calibration curves were used to convert the $\mathrm{BCA} / \mathrm{CM}$ chromatographic area to the concentration. The plasma BCA concentration versus time plot was obtained and analyzed for pharmacokinetic parameters.

\section{Chromatographic and mass spectrometry settings}

A Waters Acquity UPLC System (Waters Co.) was used for chromatographic analysis, consisting of a binary pump solvent management system, an online degasser, and an autosampler. Chromatographic separation was carried out at $30^{\circ} \mathrm{C}$ on a reversed-phase $\mathrm{C}_{18}$ column $(100 \times 2.1 \mathrm{~mm}, 1.7 \mu \mathrm{m})$. The mobile phase, pumped at $0.3 \mathrm{~mL} / \mathrm{min}$, was composed of $\mathrm{A}$ (acetonitrile) and $\mathrm{B}$ (water) using a gradient elution of $38 \% \mathrm{~A}$ at $0-3 \mathrm{~min}, 100 \% \mathrm{~A}$ at $3.2-4.2 \mathrm{~min}$, and $38 \% \mathrm{~A}$ at 4.4-5.0 min. The injection volume was $5 \mu \mathrm{L}$.

Mass spectrometry was carried out on an electrospray ionization source in the negative mode. The parameters of the source were set as follows: source temperature, $150^{\circ} \mathrm{C}$; desolvation temperature, $500^{\circ} \mathrm{C}$; cone gas flow, $50 \mathrm{~L} / \mathrm{h}$; desolvation gas flow, 1,000 L/h; capillary voltage, $3.3 \mathrm{kV}$. The dwell time was automatically set by the software. Analytic detection was performed using the multiple reaction monitoring mode at $\mathrm{m} / \mathrm{z}$ transitions $282.77 \rightarrow 267.84$ for BCA, and $320.77 \rightarrow 151.81$ for CM.

\section{Statistical analysis}

Data are expressed as the mean \pm standard deviation of at least three experiments. In all cases, $P<0.05$ was considered statistically significant. A Student's $t$-test was used to determine statistically significant differences between the experimental groups. Data analysis was performed using Statistical Package for the Social Sciences (SPSS) 18.0 (SPSS, Armonk, NY, USA).

\section{Results and discussion Physicochemical characterization}

A range of single-factor experiments was performed to investigate the impact of different proportions of F127 and S630 on the preparation of micelles. The micelles showed beneficial properties at a ratio of $1: 1$ (400 mg 


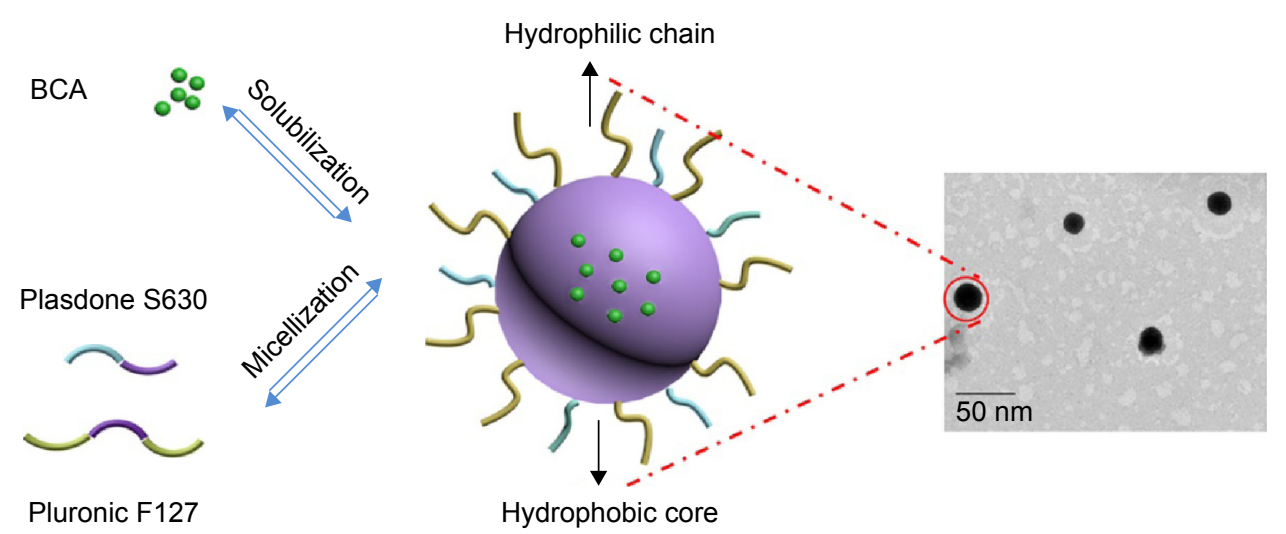

Figure 2 Structural representation and transmission electron microscopy image of BCA-loaded FI27/S630 mixed polymeric micelles. Abbreviations: BCA, biochanin A; BCA-FS, BCA-loaded micelles with Pluronic FI27 and Plasdone S630.

F127 to $400 \mathrm{mg}$ S630). The mean diameter of BCA-FS was $25.17 \pm 1.2 \mathrm{~nm}$, and the PDI was $0.147 \pm 0.011$. The zeta potential was $-10.9 \pm 0.24 \mathrm{mV}$, which could supply sufficient electrostatic repulsion between the micelles to keep them sterically stable. TEM morphology analysis revealed that the structure of BCA-FS was spherical and homogeneous (Figure 2). The water-insoluble drug could remain in the core, which was composed of hydrophobic segments and surrounded by a hydrophilic chain. Hence, the mixed micelles could significantly increase the solubility of BCA, reaching $5.0 \mathrm{mg} / \mathrm{mL}$, an increase of $\sim 10$ thousand times. The BCA drug-loading and EE were $5.88 \% \pm 0.76 \%$ and $93.67 \% \pm 0.98 \%$, respectively. These results show that a micelle system comprising F127 and S630 (1:1) can effectively deliver BCA, thereby improving its bioavailability (Figure 3).

\section{In vitro drug release}

$\mathrm{BCA}$ and $\mathrm{BCA}-\mathrm{FS}$ release in vitro was investigated using the dialysis method under sink conditions. The plot of accumulated release rate versus time is shown in Figure 4. Free BCA and BCA-FS micelles showed different dissolution behaviors in PBS. Free BCA was released rapidly, and $\sim 66.84 \% \pm 6.68 \%$ of BCA had been released within the initial $8 \mathrm{~h}$. Compared with free BCA, the amount released from BCA-FS micelles showed the relative burst drug release of $\mathrm{BCA}$ at the initial stage, followed by a slow release phase, which achieved $36.09 \% \pm 5.41 \%$ at $8 \mathrm{~h}$ and $72.86 \% \pm 7.28 \%$ at $72 \mathrm{~h}$. The BCA burst release from the mixed micelles may be a result of rapid disruption of the micellar system due to cohesion, a higher concentration gradient, and sink conditions in the system. ${ }^{28}$ BCA-FS showed a better sustained-release property than BCA.

\section{Transport in Caco-2 cell monolayers}

The Caco-2 cell culture model is recognized by the US Food and Drug Administration as a viable model for human intestinal absorption. In this study, BCA and BCA-FS transport in both AP-to-BL and BL-to-AP directions was investigated. TEER values $\left(>400 \Omega / \mathrm{cm}^{2}\right)$ were measured before and after transport experiments to ensure that the Caco- 2 cell-seeded
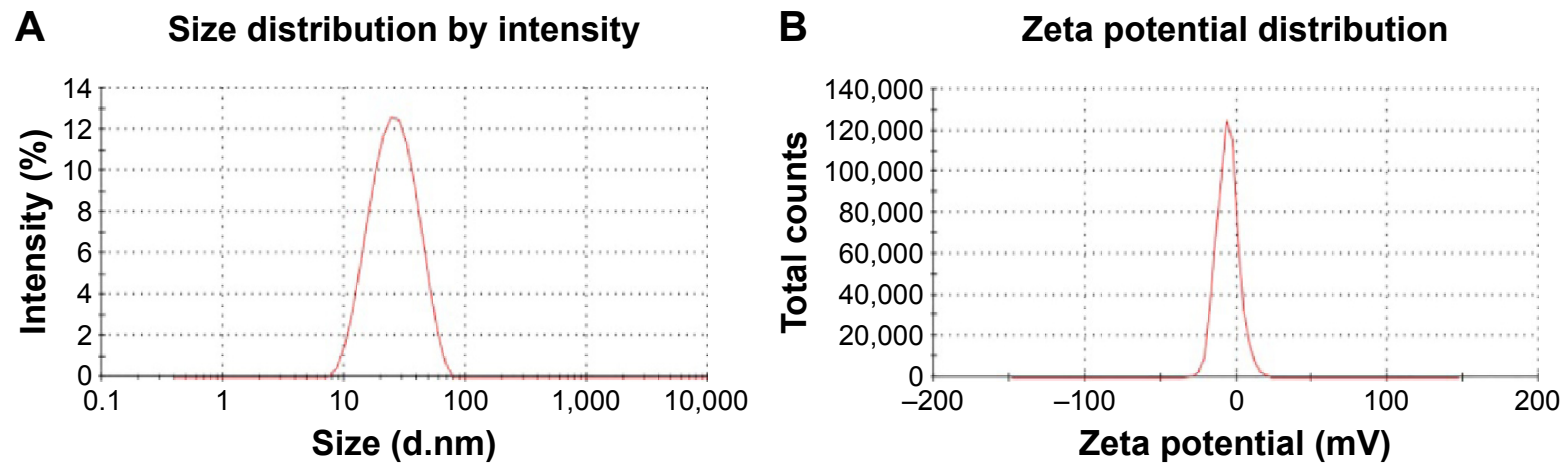

Figure 3 Size and zeta potential of BCA-FS

Notes: (A) Size distribution spectrum of BCA-FS and (B) zeta potential spectrum of BCA-FS.

Abbreviation: BCA-FS, biochanin A-loaded micelles with Pluronic FI27 and Plasdone S630. 


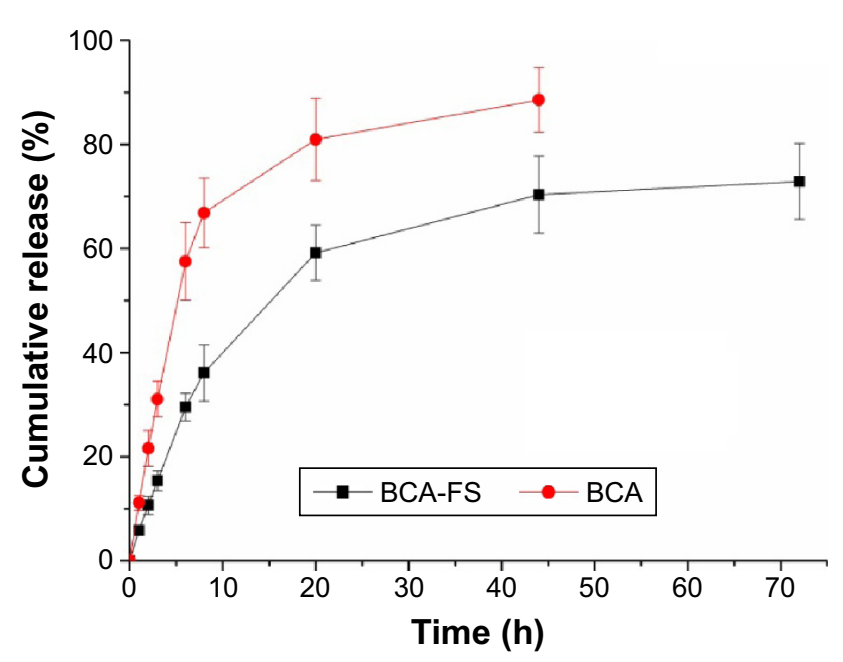

Figure 4 In vitro drug release profile of BCA-FS micelles and free BCA. Release medium, phosphate-buffered saline $(\mathrm{pH} 6.8)$ with $0.5 \%(w / v)$ Tween $80 ; n=3$.

Abbreviation: BCA-FS, biochanin A-loaded micelles with Pluronic FI27 and Plasdone S630.

inserts were integrated, and there was no toxicity to the cells. As shown in Figure 5 and Table 1, for AP-BL transport, BCA-FS significantly increased the absorption of BCA $(P<0.05)$, and the apparent permeability $\left(\mathrm{P}_{\text {app }}\right)$ value was $4.33 \pm 0.27 \times 10^{-6} \mathrm{~cm} / \mathrm{s}$, which is higher than that of the BCA group $\left(2.81 \pm 0.26 \times 10^{-6} \mathrm{~cm} / \mathrm{s}\right)$. This may be due primarily to the characteristics of Pluronic F127, which incorporates into membranes followed by subsequent translocation into cells, affecting various cellular functions. BCA transport from the $\mathrm{BL}$ to the AP side displayed no significant differences between the experimental groups. The BCA and BCA-FS $\mathrm{P}_{\text {app }}$ values were $4.58 \pm 0.33 \times 10^{-6}$ and $4.13 \pm 0.12 \times 10^{-6} \mathrm{~cm} / \mathrm{s}$,

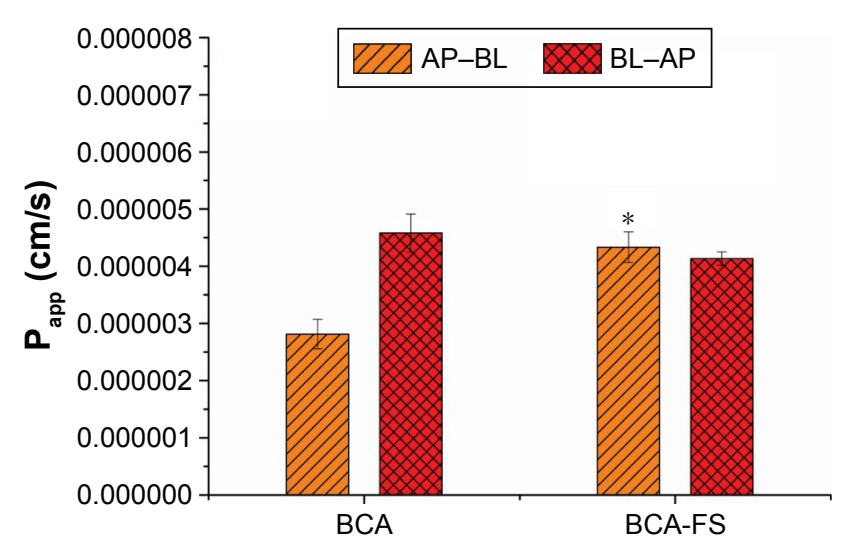

Figure 5 Permeability of BCA and BCA-FS across the Caco- 2 cell monolayer after a 4-h incubation at $37^{\circ} \mathrm{C}$.

Notes: Data are expressed as mean \pm standard deviation $(n=3)$. $* P<0.05$ versus BCA group.

Abbreviations: AP, apical; BCA, biochanin A; BL, basolateral; BCA-FS, BCA-loaded micelles with Pluronic FI27 and S630; $P_{\text {app }}$, apparent permeability.
Table I Permeability and efflux ratios

\begin{tabular}{llll}
\hline Compounds & \multicolumn{2}{l}{$\mathbf{P}_{\text {app }} \times 10^{-6}(\mathbf{c m} / \mathbf{s})$} & Efflux ratio \\
\cline { 2 - 3 } & AP-BL & BL-AP & \\
\hline BCA & $2.81 \pm 0.26$ & $4.58 \pm 0.33$ & 1.63 \\
BCA-FS & $4.33 \pm 0.27^{*}$ & $4.13 \pm 0.12$ & $0.96 *$ \\
\hline
\end{tabular}

Notes: Absorption permeability was expressed as AP-to-BL, while secretory permeability was presented as BL-to-AP. The efflux ratio was $P_{\text {app }}(B L-A P) / P_{\text {app }}(A P-B L)$. Data are expressed as mean \pm standard deviation $(n=3)$. $* P<0.05$ versus $B C A$ group.

Abbreviations: AP, apical; BCA, biochanin A; BCA-FS, BCA-loaded micelles with Pluronic FI27 and S630; BL, basolateral; $P_{\text {app }}$, apparent permeability.

respectively. $\mathrm{BCA}$ has been considered a P-glycoprotein inhibitor, ${ }^{29,30}$ and the potential inhibition mechanism may be a direct interaction, with BCA-stimulating P-gp ATPase. ${ }^{31,32}$ This could explain the relatively low $\mathrm{P}_{\text {app }}$ value. The mixed micelles system decreased the $\mathrm{P}_{\text {appBL-AP }}$, but not significantly. The efflux ratio was reduced from 1.63 to 0.96 , a remarkable difference between BCA and BCA-FS. This may be attributable to the ability of BCA-FS to increase intestinal absorption of BCA. The mechanism underlying this phenomenon may be directly related to the inclusion of the two excipients.

\section{Pharmacokinetic studies}

BCA mean plasma concentration-time curves in rats after oral administration of $50 \mathrm{mg} / \mathrm{kg}$ BCA and BCA-FS micelles $(n=6)$ are shown in Figure 6. A non-compartment model was used to calculate the pharmacokinetic parameters; the main parameters are summarized in Table 2.

There was a significant difference in $\mathrm{C}_{\max }, \mathrm{T}_{\max }$, and $\mathrm{AUC}_{0-\mathrm{t}}$ between the two groups. BCA treatment yielded a $\mathrm{C}_{\max }$ of

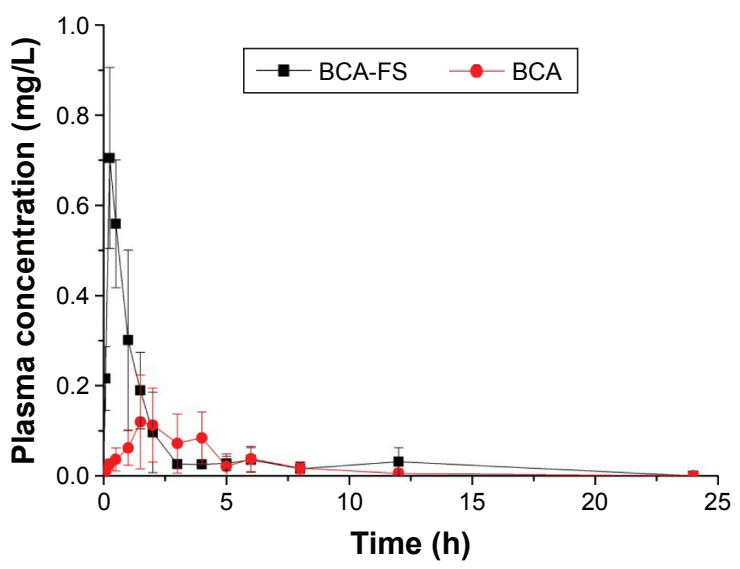

Figure 6 BCA plasma concentration-time curve in rats following an oral administration of BCA and BCA-FS $(50 \mathrm{mg} / \mathrm{kg})$.

Note: Values are expressed as mean \pm standard deviation $(n=6)$.

Abbreviations: BCA, biochanin A; BCA-FS, BCA-loaded micelles with Pluronic FI27 and Plasdone S630. 
Table 2 Main BCA pharmacokinetic parameters in rats following a $50-\mathrm{mg} / \mathrm{kg}$ oral administration of BCA and BCA-FS

\begin{tabular}{lll}
\hline Parameters & Formulations \\
\cline { 2 - 3 } & BCA & BCA-FS \\
\hline $\mathrm{AUC}_{0-\mathrm{t}}(\mathrm{mg} / \mathrm{L} \times \mathrm{h})$ & $0.524 \pm 0.239$ & $1.133 \pm 0.315^{* *}$ \\
$\mathrm{MRT}(\mathrm{h})$ & $4.114 \pm 0.864$ & $3.688 \pm 1.999$ \\
$\mathrm{t}_{1 / 2}(\mathrm{~h})$ & $0.987 \pm 0.339$ & $1.078 \pm 0.311$ \\
$\mathrm{C}_{\text {max }}(\mathrm{mg} / \mathrm{L})$ & $0.171 \pm 0.09$ & $0.785 \pm 0.218^{* *}$ \\
$\mathrm{~T}_{\text {max }}(\mathrm{h})$ & $2.4 \pm 2.04$ & $0.458 \pm 0.292^{*}$ \\
\hline
\end{tabular}

Notes: Values are expressed as mean \pm standard deviation $(n=6) . * P<0.01$, $* * P<0.05$, statistical significance versus the BCA group.

Abbreviations: $A \cup C$, area under the concentration-time curve; $B C A$, biochanin $A$; BCA-FS, BCA-loaded micelles with Pluronic $\mathrm{F} / 27$ and $\mathrm{S} 630$; $\mathrm{C}_{\text {max }}$, maximum plasma concentration; MRT, mean residence time; $T_{\text {max }}$, time to maximum plasma concentration; $\mathrm{t}_{1 / 2}$, half-life.

$0.171 \pm 0.09 \mathrm{mg} / \mathrm{L}$, which was significantly lower than the BCA-FS C $_{\text {max }}$ value of $0.785 \pm 0.218 \mathrm{mg} / \mathrm{L}(P<0.01)$. BCA-FS had a significantly higher $\mathrm{AUC}_{0-\mathrm{t}}$ of $1.133 \pm 0.315 \mathrm{mg} / \mathrm{L} / \mathrm{h}$ versus $0.524 \pm 0.239 \mathrm{mg} / \mathrm{L} / \mathrm{h}$ for BCA $(P<0.01)$, an $\sim 2.2$-fold increase in apparent bioavailability. $\mathrm{T}_{\max }$ for BCA-FS was $0.458 \mathrm{~h}$, shorter than the $\mathrm{T}_{\max }$ of $2.4 \mathrm{~h}$ for BCA, indicating that BCA-FS was rapidly digested and the BCA was absorbed upon entering the gastrointestinal tract. The $t_{1 / 2}$ values for BCA and BCA-FS were $0.987 \pm 0.339$ and $1.078 \pm 0.311 \mathrm{~h}$, respectively, showing a slightly sustained but not significant release effect in the micelles groups.
In vivo bioavailability testing further confirmed that BCA-FS increased the plasma concentration of BCA and improved its bioavailability, perhaps because of the following reasons: 1) The F127 and S630 formulation has a small diameter and large dispersion. The particle size and surface properties further affect absorption. 2) Surfactants increase intestinal epithelial permeability by disturbing the cell membrane and reversibly opening the tight junction of intestinal epithelial cells. 3) The sustained release property of BCA-FS can achieve a longer retention time in vivo. In addition, the slight P-gp inhibition and the dosage of BCA-FS could also contribute to the improvement in bioavailability. ${ }^{33}$

\section{Gastrointestinal safety assay}

We evaluated the gastrointestinal toxicity of BCA-FS in rats after intragastric administration. ${ }^{34}$ As shown in Figure 7, the stomach wall and intestinal tract showed no pathological changes after BCA treatment, and the intestinal layer possesses an explicit structure. In the BCA-FS group, the gastric wall surface appeared slightly keratinized and the intestinal villi were shortened and appeared relatively loose; there were no inflammatory cell infiltrations or cellular morphological variations. These results suggest that BCA crossed the gastrointestinal membrane easily when the drug was delivered
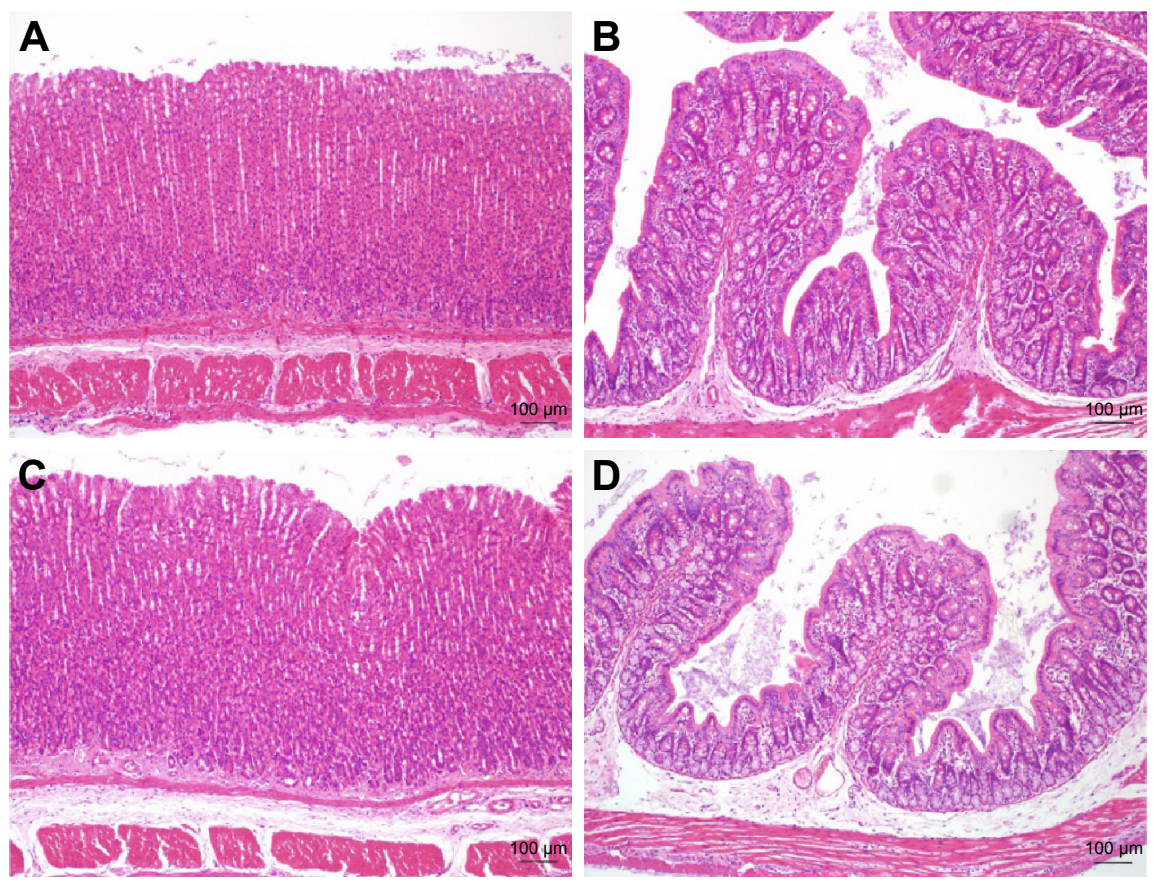

Figure 7 Gastrointestinal safety assay.

Notes: (A) Stomach tissue following BCA treatment; (B) intestinal tissue following BCA treatment; (C) stomach tissue following BCA-FS treatment; and (D) intestinal tissue following BCA-FS treatment.

Abbreviations: BCA, biochanin A; BCA-FS, BCA-loaded micelles with Pluronic FI27 and Plasdone S630. 
by the micelle mixture. Our results show that BCA-FS may stimulate the intestine mildly, but no toxicity was apparent.

\section{Conclusion}

In this study, we showed the suitability of BCA-FS as a micelle formulation for systemic delivery of BCA. BCA-FS was designed to enhance the solubility and oral absorption of BCA and showed better physical characteristics and a sustained release profile in vitro. BCA permeability across a Caco-2 cell monolayer was enhanced, and pharmacokinetic experiments in SD rats showed that the relative bioavailability of BCA-FS ( AUC $_{0-\mathrm{t}}$ ) was $216 \%$ greater than that of raw $\mathrm{BCA}$. H\&E staining revealed no acute toxicity to the gastrointestinal organs. Our findings suggest that this system, comprising Pluronic F127 and Plasdone S630, could be a promising carrier for improving the poor aqueous solubility and bioavailability of BCA.

\section{Acknowledgments}

The authors greatly acknowledge Ting Liu, Jian Hou, and Dengjie Wang for their help in pharmacokinetic studies. This work was supported by the Fundamental Research Funds for the Central Universities (021414380220) and National Natural Science Foundation of China (81403121).

\section{Disclosure}

The authors report no conflicts of interest in this work.

\section{References}

1. Szliszka E, Czuba ZP, Mertas A, Paradysz A, Krol W. The dietary isoflavone biochanin-A sensitizes prostate cancer cells to TRAIL-induced apoptosis. Urol Oncol. 2013;31(3):331-342.

2. Chen J, Ge B, Wang Y, Ye Y, Zeng S, Huang Z. Biochanin A promotes proliferation that involves a feedback loop of microRNA-375 and estrogen receptor alpha in breast cancer cells. Cell Physiol Biochem. 2015; 35(2):639-646.

3. Bhardwaj V, Tadinada SM, Jain A, et al. Biochanin A reduces pancreatic cancer survival and progression. Anticancer Drugs. 2014;25(3): 296-302.

4. Barnes S. The chemopreventive properties of soy isoflavonoids in animal models of breast cancer. Breast Cancer Res Treat. 1997;46(2-3): 169-179.

5. Kole L, Giri B, Manna SK, Pal B, Ghosh S. Biochanin-A, an isoflavone, showed anti-proliferative and anti-inflammatory activities through the inhibition of iNOS expression, p38-MAPK and ATF-2 phosphorylation and blocking NFkappaB nuclear translocation. Eur J Pharmacol. 2011;653(1-3):8-15.

6. Sithisarn P, Michaelis M, Schubert-Zsilavecz M, Cinatl J Jr. Differential antiviral and anti-inflammatory mechanisms of the flavonoids biochanin A and baicalein in H5N1 influenza A virus-infected cells. Antiviral Res. 2013;97(1):41-48.

7. Lee KH, Choi EM. Biochanin A stimulates osteoblastic differentiation and inhibits hydrogen peroxide-induced production of inflammatory mediators in MC3T3-E1 cells. Biol Pharm Bull. 2005;28(10): 1948-1953.
8. Nikander E, Metsä-Heikkilä M, Ylikorkala O, Tiitinen A. Effects of phytoestrogens on bone turnover in postmenopausal women with a history of breast cancer. J Clin Endocrinol Metab. 2004;89(3):1207-1212.

9. Harini R, Ezhumalai M, Pugalendi KV. Antihyperglycemic effect of biochanin A, a soy isoflavone, on streptozotocin-diabetic rats. Eur $J$ Pharmacol. 2012;676(1-3):89-94.

10. Jain A, Lai JC, Bhushan A. Biochanin A inhibits endothelial cell functions and proangiogenic pathways: implications in glioma therapy. Anticancer Drugs. 2015;26(3):323-330.

11. Moon YJ, Sagawa K, Frederick K, Zhang S, Morris ME. Pharmacokinetics and bioavailability of the isoflavone biochanin $\mathrm{A}$ in rats. AAPSJ. 2006;8(3):E433-E442.

12. Wang Q, Cheng H, Zhou K, et al. Nanostructured lipid carriers as a delivery system of biochanin A. Drug Deliv. 2013;20(8):331-337.

13. Wang L, Luo Q, Lin T, et al. PEGylated nanostructured lipid carriers (PEG-NLC) as a novel drug delivery system for biochanin A. Drug Dev Ind Pharm. 2015;41(7):1204-1212.

14. Attia ABE, Zhan YO, Hedrick JL, et al. Mixed micelles self-assembled from block copolymers for drug delivery. Curr Opin Colloid Interface Sci. 2011;16(3):182-194.

15. Ling G, Zhang P, Zhang W, et al. Development of novel self-assembled DS-PLGA hybrid nanoparticles for improving oral bioavailability of vincristine sulfate by P-gp inhibition. J Control Release. 2010; 148(2):241-248.

16. Song Y, Tian Q, Huang Z, et al. Self-assembled micelles of novel amphiphilic copolymer cholesterol-coupled F68 containing cabazitaxel as a drug delivery system. Int J Nanomedicine. 2014;9(1): 2307-2317.

17. Zhang W, Shi Y, Chen Y, Ye J, Sha X, Fang X. Multifunctional Pluronic P123/F127 mixed polymeric micelles loaded with paclitaxel for the treatment of multidrug resistant tumors. Biomaterials. 2011;32(11):2894-2906.

18. Zhang W, Shi Y, Chen Y, Hao J, Sha X, Fang X. The potential of Pluronic polymeric micelles encapsulated with paclitaxel for the treatment of melanoma using subcutaneous and pulmonary metastatic mice models. Biomaterials. 2011;32(25):5934-5944.

19. Wei Z, Yuan S, Chen Y, et al. Enhanced antitumor efficacy by Paclitaxelloaded Pluronic P123/F127 mixed micelles against non-small cell lung cancer based on passive tumor targeting and modulation of drug resistance. Eur J Pharm Biopharm. 2010;75(3):341-353.

20. Mitra A, Li L, Marsac P, Marks B, Zhen L, Brown C. Impact of polymer type on bioperformance and physical stability of hot melt extruded formulations of a poorly water soluble drug. Int J Pharm. 2016;505(1-2): $107-114$.

21. Maniruzzaman M, Rana MM, Boateng JS, Mitchell JC, Douroumis D. Dissolution enhancement of poorly water-soluble APIs processed by hotmelt extrusion using hydrophilic polymers. Drug Dev Ind Pharm. 2013; 39(2):218-227.

22. Ghebremeskel A, Vemavarapu C, Lodaya M. Use of surfactants as plasticizers in preparing solid dispersions of poorly soluble API: selection of polymer-surfactant combinations using solubility parameters and testing the processability. Int J Pharm. 2007;328(2):119-129.

23. Hou J, Wang J, Sun E, et al. Preparation and evaluation of icariside II-loaded binary mixed micelles using Soluto ${ }^{\circledR}$ HS15 and Pluronic F127 as carriers. Drug Deliv. 2016;23(9):1-24.

24. Jin X, Zhang ZH, Sun E, et al. Enhanced oral absorption of 20(S)protopanaxadiol by self-assembled liquid crystalline nanoparticles containing piperine: in vitro and in vivo studies. Int J Nanomedicine. 2013; 8(1):641-652.

25. Elgart A, Cherniakov I, Aldouby Y, Domb AJ, Hoffman A. Improved oral bioavailability of BCS class 2 compounds by self nano-emulsifying drug delivery systems (SNEDDS): the underlying mechanisms for amiodarone and talinolol. Pharm Res. 2013;30(12):3029-3044.

26. Agüeros M, Zabaleta V, Espuelas S, Campanero MA, Irache JM. Increased oral bioavailability of paclitaxel by its encapsulation through complex formation with cyclodextrins in poly(anhydride) nanoparticles. J Control Release. 2010;145(1):2-8. 
27. Dahmani FZ, Yang H, Zhou J, Yao J, Zhang T, Zhang Q. Enhanced oral bioavailability of paclitaxel in pluronic/LHR mixed polymeric micelles: preparation, in vitro and in vivo evaluation. Eur J Pharm Sci. 2012; 47(1):179-189.

28. Butt AM, Amin MCIM, Katas H, Sarisuta N, Witoonsaridsilp W, Benjakul R. In vitro characterization of pluronic F127 and D- $\alpha-$ tocopheryl polyethylene glycol 1000 succinate mixed micelles as nanocarriers for targeted anticancer-drug delivery. J Nanomater. 2012; 2012(2):11.

29. Zhang S, Sagawa K, Arnold RD, Tseng E, Wang X, Morris ME. Interactions between the flavonoid biochanin $\mathrm{A}$ and $\mathrm{P}$-glycoprotein substrates in rats: in vitro and in vivo. J Pharm Sci. 2010;99(1):430-441.

30. Peng SX, Ritchie DM, Cousineau M, Danser E, Dewire R, Floden J. Altered oral bioavailability and pharmacokinetics of P-glycoprotein substrates by coadministration of biochanin A.J Pharm Sci. 2006;95(9):1984-1993.
31. Zhang S, Morris ME. Effects of the flavonoids biochanin A, morin, phloretin, and silymarin on P-glycoprotein-mediated transport. JPharmacol Exp Ther. 2003;304(3):1258-1267.

32. Aller SG. Structure of P-glycoprotein reveals a molecular basis for poly-specific drug binding. Science. 2010;323(5922):1718-1722.

33. Li L, Ye BT. Preparation and properties of mixed micelles made of pluronic polymer and PEG-PE. J Colloid Interface Sci. 2008; 317(1):326-331.

34. Hajrezaie M, Salehen N, Karimian H, et al. Biochanin a gastroprotective effects in ethanol-induced gastric mucosal ulceration in rats. PLoS One. 2015;10(3):e0121529.
International Journal of Nanomedicine

\section{Publish your work in this journal}

The International Journal of Nanomedicine is an international, peerreviewed journal focusing on the application of nanotechnology in diagnostics, therapeutics, and drug delivery systems throughout the biomedical field. This journal is indexed on PubMed Central, MedLine, CAS, SciSearch $®$, Current Contents $\AA /$ Clinical Medicine,

\section{Dovepress}

Journal Citation Reports/Science Edition, EMBase, Scopus and the Elsevier Bibliographic databases. The manuscript management system is completely online and includes a very quick and fair peer-review system, which is all easy to use. Visit http://www.dovepress.com/ testimonials.php to read real quotes from published authors.

Submit your manuscript here: http://www.dovepress.com/international-journal-of-nanomedicine-journal 